Research Article

\title{
Nonlinear Jordan Derivable Mappings of Generalized Matrix Algebras by Lie Product Square-Zero Elements
}

\author{
Xiuhai Fei and Haifang Zhang \\ School of Mathematics and Physics, West Yunnan University, Lingcang 677099, China \\ Correspondence should be addressed to Haifang Zhang; 907427798@qq.com
}

Received 30 July 2021; Accepted 18 August 2021; Published 10 September 2021

Academic Editor: Wenpeng Zhang

Copyright (C) 2021 Xiuhai Fei and Haifang Zhang. This is an open access article distributed under the Creative Commons Attribution License, which permits unrestricted use, distribution, and reproduction in any medium, provided the original work is properly cited.

The aim of the paper is to give a description of nonlinear Jordan derivable mappings of a certain class of generalized matrix algebras by Lie product square-zero elements. We prove that under certain conditions, a nonlinear Jordan derivable mapping $\Delta$ of a generalized matrix algebra by Lie product square-zero elements is a sum of an additive derivation $\delta$ and an additive antiderivation $f$. Moreover, $\delta$ and $f$ are uniquely determined.

\section{Introduction}

Throughout the paper, by an algebra we shall mean an algebra over a fixed unital commutative ring $\mathscr{R}$. Let $\mathscr{A}$ be a unital algebra, $P$ be a fixed element of $\mathscr{A}$, $\Omega=\left\{A \in \mathscr{A}: A^{2}=0\right\}$, and $\Delta: \mathscr{A} \longmapsto \mathscr{A}$ be an additive (resp., without assumption of additivity) mapping. For any $A, B \in \mathscr{A}$, denote the Jordan product (resp., Lie product) of $A$ and $B$ by $A \circ B=\mathrm{AB}+\mathrm{BA}$ (resp., $[A, B]=\mathrm{AB}-\mathrm{BA})$. For any $A \in \mathscr{A}$, if $2 A=0$, implying $A=0$, then $\mathscr{A}$ is said to be a 2 -torsion free algebra. Recall that $\Delta$ is called an additive derivation (resp., nonlinear derivable mapping) if $\Delta(\mathrm{AB})=$ $\Delta(A) B+A \Delta(B)$ for all $A, B \in \mathscr{A}$. It is called an additive antiderivation (resp., nonlinear antiderivable mapping) if $\Delta(\mathrm{AB})=\Delta(B) A+B \Delta(A)$ for all $A, B \in \mathscr{A}$. It is called an additive Jordan derivation (resp., nonlinear Jordan derivable mapping) if $\Delta(A \circ B)=\Delta(A) \circ B+A \circ \Delta(B)$ for all $A, B \in \mathscr{A}$. It is called a $P$ point derivable mapping (resp., $P$ point nonlinear derivable mapping) if $\Delta(\mathrm{AB})=\Delta(A) B+A \Delta(B)$ for all $A, B \in \mathscr{A}$ with $\mathrm{AB}=P$. Similarly, we have the definitions of $P$ point Jordan derivable mapping and $P$ point nonlinear Jordan derivable mapping. In this paper, if $\Delta$ is without assumption of additivity and satisfies

$$
\Delta(A \circ B)=\Delta(A) \circ B+A \circ \Delta(B),
$$

for all $A, B \in \mathscr{A}$ with $[A, B] \in \Omega$, then we say $\Delta$ is a nonlinear Jordan derivable mapping of $\mathscr{A}$ by Lie product square-zero elements. Obviously, every additive derivation or additive antiderivation is an additive Jordan derivation. However, the inverse statement is not true in general (see [1]).

A natural and very interesting problem that we are dealing with is studying those conditions on a ring or an algebra such that every additive Jordan derivation or every nonlinear Jordan derivable map is an additive derivation.

In the past few decades, the research on this problem has attracted the attention of many mathematicians. For examples, Herstein in [2] proved that every additive Jordan derivation on a prime ring not of characteristic 2 is an additive derivation. Later, this result was extended by Cusack in [3] and Brešar and Vukman in [4] to the case of semiprime ring, respectively. Zhang and Yu in $[5,6]$ showed that every additive Jordan derivation on a nest algebra and a 2-torsion free triangular algebra is an inner derivation and an additive derivation, respectively. For some conclusions about $P$ point Jordan derivable mapping, we refer the readers to [7-9] and references therein for more details. $\mathrm{Lu}$ in [10] showed that every nonlinear Jordan derivable mapping on a 2-torsion free semiprime ring is an additive derivation. Ashraf and Jabeen in [11] showed that every nonlinear Jordan triple derivable mapping on a 2-torsion 
free triangular algebra is an additive derivation. In particular, Benkovič in [1] proved that every additive Jordan derivation from an upper triangular matrix algebra to its bimodule is a sum of an additive derivation and an additive antiderivation. Li et al. in [12] proved that under certain conditions every additive Jordan derivation on a generalized matrix algebra is a sum of an additive derivation and an additive antiderivation. For other similar results, we refer the readers to $[13,14]$ and references therein for more details.

Inspired by the above references, in this paper, we study the nonlinear Jordan derivable mapping of generalized matrix algebras by Lie product square-zero elements, we get that under certain conditions, a nonlinear Jordan derivable mapping of a generalized matrix algebra by Lie product square-zero elements is a sum of an additive derivation and an additive antiderivation.

\section{Generalized Matrix Algebras}

Let $\mathscr{A}$ and $\mathscr{B}$ be two unital algebras with unit elements $1_{\mathscr{A}}$ and $1_{\mathscr{B}}$, respectively, $\mathscr{M}$ be a faithful $(\mathscr{A}, \mathscr{B})$-bimodule, and $\mathcal{N}$ be a $(\mathscr{B}, \mathscr{A})$-bimodule. Then,

$$
\mathscr{G}=\left(\begin{array}{cc}
\mathscr{A} & \mathscr{M} \\
\mathcal{N} & \mathscr{B}
\end{array}\right)=\left\{\left(\begin{array}{cc}
A & M \\
N & B
\end{array}\right): A \in \mathscr{A}, M \in \mathscr{M}, N \in \mathcal{N}, B \in \mathscr{B}\right\}
$$

is a $\mathscr{R}$-algebra under matrix-like addition and multiplication, satisfying the following commutative diagrams:
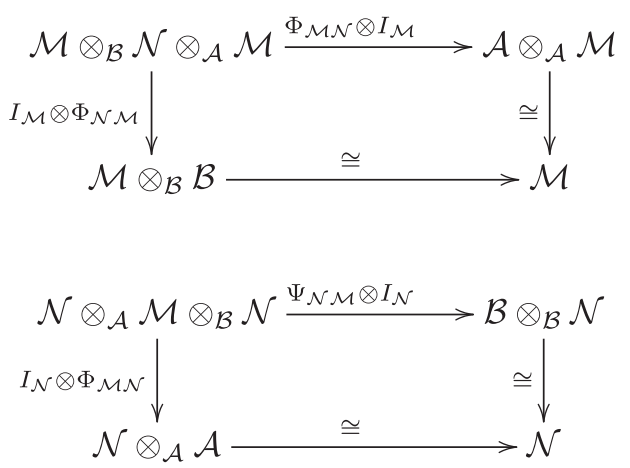

where $\Phi_{\mathscr{M}}: \mathscr{M}_{\mathscr{B}} \mathcal{N} \longmapsto \mathscr{A}$ and $\Psi_{\mathscr{N} \mathscr{M}}: \mathscr{N} \otimes_{\mathscr{A}} \mathscr{M}_{\mathfrak{B}} \longmapsto \mathscr{B}$ are two bimodule homomorphisms. Such a $\mathscr{R}$-algebra $\mathscr{G}$ is called a generalized matrix algebra. For convenience in reading, we give the multiplication of generalized matrix algebra as follows:

$$
\left(\begin{array}{cc}
A & M \\
N & B
\end{array}\right)\left(\begin{array}{cc}
A^{\prime} & M^{\prime} \\
N^{\prime} & B^{\prime}
\end{array}\right)=\left(\begin{array}{cc}
A A^{\prime}+\Phi_{\mathscr{M N}}\left(M \otimes N^{\prime}\right) & A M^{\prime}+M B^{\prime} \\
N A^{\prime}+B N^{\prime} & B B^{\prime}+\Psi_{\mathcal{N} \mathscr{M}}\left(N \otimes M^{\prime}\right)
\end{array}\right)
$$

for all $A, A^{\prime} \in \mathscr{A}, M, M^{\prime} \in \mathscr{M}, N, N^{\prime} \in \mathcal{N}$, and $B, B^{\prime} \in \mathscr{B}$. Furthermore, when $\mathscr{N}=0$, then $\mathscr{G}$ is a triangular algebra. The most common examples of generalized matrix algebras are triangular algebras and full matrix algebras (see $[15,16]$ for details).

Considering a generalized matrix algebra $\mathscr{G}$, let 1 be the unit element of $\mathscr{G}$. Set

$$
\begin{array}{r}
P_{1}=\left(\begin{array}{cc}
1_{\mathscr{A}} & 0 \\
0 & 0
\end{array}\right), \\
P_{2}=1-P_{1}=\left(\begin{array}{cc}
0 & 0 \\
0 & 1_{\mathscr{B}}
\end{array}\right),
\end{array}
$$

and $\mathscr{G}_{i j}=P_{i} \mathscr{G} P_{j}(1 \leq i, j \leq 2)$. It is clear that if $\Phi_{\mathscr{M N}}\left(M \otimes N^{\prime}\right)=0=\Psi_{\mathscr{N} \mathscr{M}}\left(N \otimes M^{\prime}\right)$, then $\mathscr{G}$ can be represented as

$$
\mathscr{G}=\mathscr{A}+\mathscr{M}+\mathscr{N}+\mathscr{B}=\mathscr{G}_{11}+\mathscr{G}_{12}+\mathscr{G}_{21}+\mathscr{G}_{22} .
$$

Hence, for any $A \in \mathscr{G}, A$ can be represented as $A=A_{11}+A_{12}+A_{21}+A_{22}$, where $A_{i j} \in \mathscr{G}_{i j}(1 \leq i, j \leq 2)$, and we can easily check that $A_{i j} B_{j i}=0, \forall A_{i j} \in \mathscr{G}_{i j}$, and $B_{j i} \in \mathscr{G}_{j i}(1 \leq i \neq j \leq 2)$ (i.e., $\left.\mathscr{M} \mathscr{N}=\mathscr{N} \mathscr{M}=0\right)$.

\section{Main Results}

In this paper, our main result is the following theorem.

Theorem 1. Let $\mathscr{G}$ be a 2-torsion free generalized matrix algebra and $\Delta$ be a nonlinear Jordan derivable mapping of $\mathscr{G}$ by Lie product square-zero elements. If $\mathscr{G}$ satisfies $\mathscr{M} \mathscr{N}=\mathscr{N} \mathscr{M}=0$, then there exist an additive derivation $\delta$ and an additive antiderivation $f$ of $\mathscr{G}$, such that $\Delta(A)=\delta(A)+f(A)$ for all $A \in \mathscr{G}$. Moreover, $\delta$ and $f$ are uniquely determined.

In order to prove Theorem 1, we introduce some lemmas. Next, we assume that $\mathscr{G}$ is a 2 -torsion generalized matrix algebra, $\mathscr{M}$ is a faithful $(\mathscr{A}, \mathscr{B})$-bimodule and $\mathscr{N}$ is a $(\mathscr{B}, \mathscr{A})$-bimodule (i.e., $\mathscr{G}_{12}$ is a faithful $\left(\mathscr{G}_{11}, \mathscr{G}_{22}\right)$-bimodule and $\mathscr{G}_{21}$ is a $\left(\mathscr{G}_{22}, \mathscr{G}_{11}\right)$-bimodule), $\mathscr{M} \mathscr{N}=\mathscr{N} \mathscr{M}=0$ (i.e., $A_{i j} B_{j i}=0, \forall A_{i j} \in \mathscr{G}_{i j}$, and $\left.B_{j i} \in \mathscr{G}_{j i}(1 \leq i \neq j \leq 2)\right)$, 
$\Omega=\left\{A \in \mathscr{G}: A^{2}=0\right\}$, and $\Delta$ is a nonlinear Jordan derivable mapping of $\mathscr{G}$ by Lie product square-zero elements.

Lemma 1. For any $A_{i i} \in \mathscr{G}_{i i}, A_{i j}, B_{i j} \in \mathscr{G}_{i j}, \quad C_{j i} \in \mathscr{G}_{j i}$ $(1 \leq i \neq j \leq 2)$, then

(i) $\Delta(0)=0$

(ii) $\Delta\left(P_{i}\right)=P_{i} \Delta\left(P_{i}\right) P_{j}+P_{j} \Delta\left(P_{i}\right) P_{i}, \quad \Delta\left(P_{1}\right)=-\Delta\left(P_{2}\right)$, and $A_{i j} \circ \Delta\left(P_{1}\right)=A_{i j} \circ \Delta\left(P_{2}\right)=0$

(iii) $\Delta\left(A_{i j}\right)=P_{i} \Delta\left(A_{i j}\right) P_{j}+P_{j} \Delta\left(A_{i j}\right) P_{i}$ and $A_{i j} \circ \Delta\left(B_{i j}\right)$ $=A_{i j} \circ \Delta\left(C_{j i}\right)=0$

(iv) $P_{j} \Delta\left(A_{i i}\right) P_{j}=0, P_{i} \Delta\left(A_{i i}\right) P_{j}=A_{i i} \Delta\left(P_{i}\right)$ and $P_{j} \Delta$ $\left(A_{i i}\right) P_{i}=\Delta\left(P_{i}\right) A_{i i}$

Proof

(i) Since $\Delta$ is a nonlinear Jordan derivable mapping of $\mathscr{G}$ by Lie product square-zero elements, then for any $A, B \in \mathscr{G}$ with $[A, B] \in \Omega$, we have

$$
\Delta(A \circ B)=\Delta(A) \circ B+A \circ \Delta(B) .
$$

Therefore, taking $A=B=0$ in equation (8), we have $\Delta(0)=\Delta(0) \circ 0+0 \circ \Delta(0)=0$.

(ii) Since $\left[P_{i}, P_{j}\right]=0 \in \Omega(1 \leq i \neq j \leq 2)$ and taking $A=$ $P_{i}$ and $B=P_{j}$ in equation (8), we have

$0=\Delta\left(P_{i} \circ P_{j}\right)=\Delta\left(P_{i}\right) P_{j}+P_{j} \Delta\left(P_{i}\right)+P_{i} \Delta\left(P_{j}\right)+\Delta\left(P_{j}\right) P_{i}$.

Multiplying equation (9) from both the sides by $P_{j}$ and then by the property of 2 -torsion freeness of $\mathscr{G}$, we have

$$
P_{j} \Delta\left(P_{i}\right) P_{j}=0
$$

Multiplying equation (9) from the left by $P_{i}$ and from the right by $P_{j}$, we get

$$
P_{i} \Delta\left(P_{i}\right) P_{j}=-P_{i} \Delta\left(P_{j}\right) P_{j} .
$$

Next, we show that $P_{i} \Delta\left(P_{i}\right) P_{i}=0 \quad(1 \leq i \leq 2)$. Indeed, for any $X_{12} \in \mathscr{G}_{12}$, since $\left[P_{1}, X_{12}\right]=X_{12} \in \Omega$ and taking $A=P_{1}$ and $B=X_{12}$ in equation (8), we obtain

$$
\begin{aligned}
\Delta\left(X_{12}\right)= & \Delta\left(P_{1} \circ X_{12}\right) \\
= & \Delta\left(P_{1}\right) \circ X_{12}+P_{1} \circ \Delta\left(X_{12}\right) \\
= & \Delta\left(P_{1}\right) X_{12}+X_{12} \Delta\left(P_{1}\right)+P_{1} \Delta\left(X_{12}\right) \\
& +\Delta\left(X_{12}\right) P_{1} .
\end{aligned}
$$

Multiplying equation (12) from the left by $P_{1}$ and from the right by $P_{2}$ and then by equation (10), we have $P_{1} \Delta\left(P_{1}\right) X_{12}=P_{1} \Delta\left(P_{1}\right) P_{1} X_{12}=0$; similarly, we can get that $X_{12} \Delta\left(P_{2}\right) P_{2}=X_{12} P_{2} \Delta\left(P_{2}\right) P_{2}=0$; therefore, we obtain from the faithfulness of $\mathscr{G}_{12}$ that

$$
\begin{aligned}
& P_{1} \Delta\left(P_{1}\right) P_{1}=0, \\
& P_{2} \Delta\left(P_{2}\right) P_{2}=0 .
\end{aligned}
$$

Thus, we obtain from equations (10)-(12) that $\Delta\left(P_{i}\right)=P_{i} \Delta\left(P_{i}\right) P_{j}+P_{j} \Delta\left(P_{i}\right) P_{i} \quad(1 \leq i \neq j \leq 2)$ and $\Delta\left(P_{1}\right)=-\Delta\left(P_{2}\right)$, and then, it follows from $\mathscr{M} \mathscr{N}=$ $\mathscr{N} \mathscr{M}=0$ that $A_{i j} \circ \Delta\left(P_{1}\right)=A_{i j} \circ \Delta\left(P_{2}\right)=0$ for all $A_{i j} \in \mathscr{G}_{i j}(1 \leq i \neq j \leq 2)$.

(iii) For any $A_{i j} \in \mathscr{G}_{i j}$ and $C_{j i} \in \mathscr{G}_{j i}(1 \leq i \neq j \leq 2)$, since $\left[P_{i}, A_{i j}\right]=A_{i j} \in \Omega$ and taking $A=P_{i}$ and $B=A_{i j}$ in equation (8), it follows from Lemma 1 (ii) that

$$
\Delta\left(A_{i j}\right)=\Delta\left(P_{i} \circ A_{i j}\right)=\Delta\left(P_{i}\right) \circ A_{i j}+P_{i} \circ \Delta\left(A_{i j}\right)=P_{i} \Delta\left(A_{i j}\right)+\Delta\left(A_{i j}\right) P_{i} .
$$

Multiplying (14) by $P_{i}$ and $P_{j}$ from both sides, respectively, we get that

$$
P_{j} \Delta\left(A_{i j}\right) P_{j}=P_{i} \Delta\left(A_{i j}\right) P_{i}=0 .
$$

This implies that $\Delta\left(A_{i j}\right)=P_{i} \Delta\left(A_{i j}\right) P_{j}+P_{j} \Delta\left(A_{i j}\right) P_{i}$ $(1 \leq i \neq j \leq 2)$, and then by $\mathscr{M} \mathscr{N}=\mathscr{N} \mathscr{M}=0$, we get $A_{i j} \circ \Delta\left(B_{i j}\right)=A_{i j} \circ \Delta\left(C_{j i}\right)=0$.

(iv) For any $A_{i i} \in \mathscr{G}_{i i}(1 \leq i \leq 2)$, since $\left[P_{j}, A_{i i}\right]=0 \in \Omega$ $(1 \leq i \neq j \leq 2)$ and taking $A=P_{j}$ and $B=A_{i i}$ in equation (8), we get

$$
0=\Delta\left(P_{j} \circ A_{i i}\right)=\Delta\left(P_{j}\right) A_{i i}+A_{i i} \Delta\left(P_{j}\right)+P_{j} \Delta\left(A_{i i}\right)+\Delta\left(A_{i i}\right) P_{j} .
$$

Multiplying equation (16) by $P_{j}$ from both sides and then by the property of 2 -torsion freeness of $\mathscr{G}$, we have $P_{j} \Delta\left(A_{i i}\right) P_{j}=0$.

Multiplying equation (16) from the left by $P_{i}$ and from the right by $P_{j}$, it follows from Lemma 1 (ii) that

$$
P_{i} \Delta\left(A_{i i}\right) P_{j}=-A_{i i} \Delta\left(P_{j}\right) P_{j}=A_{i i} \Delta\left(P_{i}\right) P_{j}=A_{i i} \Delta\left(P_{i}\right) .
$$

Similarly, we can show that $P_{j} \Delta\left(A_{i i}\right) P_{i}=\Delta\left(P_{i}\right) A_{i i}$ holds. The proof is completed. 
Lemma 2. For any $A_{i i}, B_{i i} \in \mathscr{G}_{i i}, A_{i j}, B_{i j} \in \mathscr{G}_{i j}, A_{j i} \in \mathscr{G}_{j i}$, and $A_{j j} \in \mathscr{G}_{j j}(1 \leq i \neq j \leq 2)$, then

(i) $\Delta\left(A_{i i}+A_{i j}\right)=\Delta\left(A_{i i}\right)+\Delta\left(A_{i j}\right)$

(ii) $\Delta\left(A_{i j}+A_{j j}\right)=\Delta\left(A_{i j}\right)+\Delta\left(A_{j j}\right)$

(iii) $\Delta\left(A_{i j}+B_{i j}\right)=\Delta\left(A_{i j}\right)+\Delta\left(B_{i j}\right)$

(iv) $\Delta\left(A_{i j}+A_{j i}\right)=\Delta\left(A_{i j}\right)+\Delta\left(A_{j i}\right)$

(v) $\Delta\left(A_{i i}+B_{i i}\right)=\Delta\left(A_{i i}\right)+\Delta\left(B_{i i}\right)$

(vi) $\Delta\left(A_{i i}+A_{i j}+A_{j i}\right)=\Delta\left(A_{i i}\right)+\Delta\left(A_{i j}\right)+\Delta\left(A_{j i}\right)$ (vii) $\Delta\left(A_{i i}+A_{i j}+A_{j i}+A_{j j}\right)=\Delta\left(A_{i i}\right)+\Delta\left(A_{i j}\right)+$ $\Delta\left(A_{j i}\right)+\Delta\left(A_{j j}\right)$

Proof

(i) For any $A_{i i} \in \mathscr{G}_{i i}$ and $A_{i j} \in \mathscr{G}_{i j}(1 \leq i \neq j \leq 2)$, since $\left[A_{i i}+A_{i j}, P_{j}\right]=A_{i j} \in \Omega$, taking $A=A_{i i}+A_{i j}$ and $B=P_{j}$ in equation (8), and so by Lemma 1 (ii)-(iv), we have

$$
\begin{aligned}
\Delta\left(A_{i j}\right) & =\Delta\left(\left(A_{i i}+A_{i j}\right) \circ P_{j}\right) \\
& =\Delta\left(A_{i i}+A_{i j}\right) \circ P_{j}+\left(A_{i i}+A_{i j}\right) \circ \Delta\left(P_{j}\right) \\
& =\Delta\left(A_{i i}+A_{i j}\right) P_{j}+P_{j} \Delta\left(A_{i i}+A_{i j}\right)+A_{i i} \Delta\left(P_{j}\right)+\Delta\left(P_{j}\right) A_{i i} \\
& =\Delta\left(A_{i i}+A_{i j}\right) P_{j}+P_{j} \Delta\left(A_{i i}+A_{i j}\right)-P_{i} \Delta\left(A_{i i}\right) P_{j}-P_{j} \Delta\left(A_{i i}\right) P_{i} .
\end{aligned}
$$

This yields that

$$
\Delta\left(A_{i j}\right)+P_{i} \Delta\left(A_{i i}\right) P_{j}+P_{j} \Delta\left(A_{i i}\right) P_{i}=\Delta\left(A_{i i}+A_{i j}\right) P_{j}+P_{j} \Delta\left(A_{i i}+A_{i j}\right) .
$$

Multiplying equation (19) by $P_{j}$ from both sides and then by the property of 2-torsion freeness of $\mathscr{G}$ and Lemma 1 (iii), we have

$$
P_{j} \Delta\left(A_{i i}+A_{i j}\right) P_{j}=0
$$

Multiplying equation (19) from the left by $P_{i}$ and from the right by $P_{j}$, we get

$$
P_{i} \Delta\left(A_{i i}+A_{i j}\right) P_{j}=P_{i} \Delta\left(A_{i i}\right) P_{j}+P_{i} \Delta\left(A_{i j}\right) P_{j} .
$$

Multiplying equation (19) from the left by $P_{j}$ and from the right by $P_{i}$, we get

$$
P_{j} \Delta\left(A_{i i}+A_{i j}\right) P_{i}=P_{j} \Delta\left(A_{i i}\right) P_{i}+P_{j} \Delta\left(A_{i j}\right) P_{i} .
$$

Next, we show that $P_{i} \Delta\left(A_{i i}+A_{i j}\right) P_{i}=P_{i} \Delta\left(A_{i i}\right) P_{i}$. Indeed, for any $A_{11} \in \mathscr{G}_{11}, A_{12}, X_{12} \in \mathscr{G}_{12}$, since $\left[A_{11}+A_{12}, X_{12}\right]=\left[A_{11}, X_{12}\right]=A_{11} X_{12} \in \Omega$, on the one hand, taking $A=A_{11}+A_{12}, B=X_{12}$ in equation (8), and then by Lemma 1 (iii), we get

$$
\begin{aligned}
\Delta\left(A_{11} X_{12}\right) & =\Delta\left(\left(A_{11}+A_{12}\right) \circ X_{12}\right) \\
& =\Delta\left(A_{11}+A_{12}\right) \circ X_{12}+\left(A_{11}+A_{12}\right) \circ \Delta\left(X_{12}\right) \\
& =\Delta\left(A_{11}+A_{12}\right) X_{12}+X_{12} \Delta\left(A_{11}+A_{12}\right)+A_{11} \Delta\left(X_{12}\right)+\Delta\left(X_{12}\right) A_{11} .
\end{aligned}
$$

On the other hand, taking $A=A_{11}$ and $B=X_{12}$ in equation (8), we get that

$$
\begin{aligned}
\Delta\left(A_{11} X_{12}\right) & =\Delta\left(A_{11} \circ X_{12}\right) \\
& =\Delta\left(A_{11}\right) \circ X_{12}+A_{11} \circ \Delta\left(X_{12}\right) \\
& =\Delta\left(A_{11}\right) X_{12}+X_{12} \Delta\left(A_{11}\right)+A_{11} \Delta\left(X_{12}\right)+\Delta\left(X_{12}\right) A_{11} .
\end{aligned}
$$


Comparing equations (23) and (24), we get

$$
\left(\Delta\left(A_{11}+A_{12}\right)-\Delta\left(A_{11}\right)\right) X_{12}+X_{12}\left(\Delta\left(A_{11}+A_{12}\right)-\Delta\left(A_{11}\right)\right)=0 .
$$

Multiplying equation (25) from the left by $P_{1}$ and from the right by $P_{2}$, then by equation (20) and Lemma 1 (iv), we get

$$
P_{1}\left(\Delta\left(A_{11}+A_{12}\right)-\Delta\left(A_{11}\right)\right) P_{1} X_{12}=0 .
$$

Similarly, for any $X_{12} \in \mathscr{G}_{12}, A_{21} \in \mathscr{G}_{21}$, and $A_{22} \in \mathscr{G}_{22}$, we can get that

$$
X_{12} P_{2}\left(\Delta\left(A_{22}+A_{21}\right)-\Delta\left(A_{22}\right)\right) P_{2}=0 .
$$

This yields from the faithfulness of $\mathscr{G}_{12}$ that

$$
P_{1} \Delta\left(A_{11}+A_{12}\right) P_{1}=P_{1} \Delta\left(A_{11}\right) P_{1} \text { and } P_{2} \Delta\left(A_{22}+A_{21}\right) P_{2}=P_{2} \Delta\left(A_{22}\right) P_{2} \text {. }
$$

Therefore, it follows from equations (20)-(28) and Lemma 1 (iii)-(iv) that $\Delta\left(A_{i i}+A_{i j}\right)=\Delta\left(A_{i i}\right)+$ $\Delta\left(A_{i j}\right)$.

(ii) Similar to (i), we can show that (ii) holds. (iii) For any $A_{i j}, B_{i j} \in \mathscr{G}_{i j}(1 \leq i \neq j \leq 2)$, since $\left[P_{i}+A_{i j}, B_{i j}+P_{j}\right]=A_{i j}+B_{i j} \in \Omega$, taking $A=P_{i}+$ $A_{i j}, B=B_{i j}+P_{j}$ in equation (8), and so by Lemma 2 (i)-(ii) and Lemma 1 (ii)-(iv), we have

$$
\begin{aligned}
\Delta\left(A_{i j}+B_{i j}\right) & =\Delta\left(\left(P_{i}+A_{i j}\right) \circ\left(B_{i j}+P_{j}\right)\right) \\
& =\Delta\left(P_{i}+A_{i j}\right) \circ\left(B_{i j}+P_{j}\right)+\left(P_{i}+A_{i j}\right) \circ \Delta\left(B_{i j}+P_{j}\right) \\
& =\left(\Delta\left(P_{i}\right)+\Delta\left(A_{i j}\right)\right) \circ\left(B_{i j}+P_{j}\right)+\left(P_{i}+A_{i j}\right) \circ\left(\Delta\left(B_{i j}\right)+\Delta\left(P_{j}\right)\right) \\
& =\Delta\left(P_{i}\right) \circ P_{j}+\Delta\left(A_{i j}\right) \circ P_{j}+P_{i} \circ \Delta\left(B_{i j}\right)+P_{i} \circ \Delta\left(P_{j}\right) \\
& =\Delta\left(P_{i}\right)+\Delta\left(A_{i j}\right)+\Delta\left(B_{i j}\right)+\Delta\left(P_{j}\right) \\
& =\Delta\left(A_{i j}\right)+\Delta\left(B_{i j}\right)
\end{aligned}
$$

(iv) For any $A_{i j} \in \mathscr{G}_{i j}$ and $A_{j i} \in \mathscr{G}_{i i}(1 \leq i \neq j \leq 2)$, it follows from $\mathscr{M} \mathscr{N}=\mathcal{N} \mathscr{M}=0$ that $\left[P_{j}+A_{i j}, A_{j i}+\right.$ $\left.P_{i}\right]=-A_{i j}+A_{i j} A_{j i}-A_{j i} A_{i j}+A_{j i}=-A_{i j}+A_{i j} \in \Omega$; taking $A=P_{j}+A_{i j}$ and $B=A_{j i}+P_{i}$ in equation (8) and we get from $\mathscr{M} \mathcal{N}=\mathscr{N} \mathscr{M}=0$, Lemma 2 (i)-(ii), and Lemma 1 (ii)-(iv) that

$$
\begin{aligned}
\Delta\left(A_{i j}+A_{j i}\right) & =\Delta\left(\left(P_{j}+A_{i j}\right) \circ\left(A_{j i}+P_{i}\right)\right) \\
& =\Delta\left(P_{j}+A_{i j}\right) \circ\left(A_{j i}+P_{i}\right)+\left(P_{j}+A_{i j}\right) \circ \Delta\left(A_{j i}+P_{i}\right) \\
& =\left(\Delta\left(P_{j}\right)+\Delta\left(A_{i j}\right)\right) \circ\left(A_{j i}+P_{i}\right)+\left(P_{j}+A_{i j}\right) \circ\left(\Delta\left(A_{j i}\right)+\Delta\left(P_{i}\right)\right) \\
& =\Delta\left(P_{j}\right) \circ P_{i}+\Delta\left(A_{i j}\right) \circ P_{i}+P_{j} \circ \Delta\left(A_{j i}\right)+P_{j} \circ \Delta\left(P_{i}\right) \\
& =\Delta\left(P_{j}\right)+\Delta\left(A_{i j}\right)+\Delta\left(A_{j i}\right)+\Delta\left(P_{i}\right) \\
& =\Delta\left(A_{i j}\right)+\Delta\left(A_{j i}\right) .
\end{aligned}
$$


(v) For any $A_{11}, B_{11} \in \mathscr{G}_{11}$, it follows from Lemma 1 (iv) that

$$
\begin{gathered}
\Delta\left(A_{11}\right)=P_{1} \Delta\left(A_{11}\right) P_{1}+A_{11} \Delta\left(P_{1}\right)+\Delta\left(P_{1}\right) A_{11}, \\
\Delta\left(B_{11}\right)=P_{1} \Delta\left(B_{11}\right) P_{1}+B_{11} \Delta\left(P_{1}\right)+\Delta\left(P_{1}\right) B_{11}, \\
\Delta\left(A_{11}+B_{11}\right)=P_{1} \Delta\left(A_{11}+B_{11}\right) P_{1}+\left(A_{11}+B_{11}\right) \Delta\left(P_{1}\right)+\Delta\left(P_{1}\right)\left(A_{11}+B_{11}\right) .
\end{gathered}
$$

In the following, we show that $P_{1} \Delta\left(A_{11}+B_{11}\right) P_{1}=P_{1} \Delta\left(A_{11}\right) P_{1}+P_{1} \Delta\left(B_{11}\right) P_{1}$. Indeed, for any $A_{11}, B_{11} \in \mathscr{G}_{11}, X_{12} \in \mathscr{G}_{12}$, on the one hand, since $\left[A_{11}+B_{11}, X_{12}\right]=\left(A_{11}+B_{11}\right)$ $X_{12} \in \Omega$ and taking $A=A_{11}+B_{11}$ and $B=X_{12}$ in equation (8), then we get

$$
\Delta\left(\left(A_{11}+B_{11}\right) X_{12}\right)=\Delta\left(A_{11}+B_{11}\right) \circ X_{12}+\left(A_{11}+B_{11}\right) \circ \Delta\left(X_{12}\right) .
$$

On the other hand, since $\left[A_{11}, X_{12}\right]=A_{11} X_{12} \in \Omega$ and $\left[B_{11}, X_{12}\right]=B_{11} X_{12} \in \Omega$, we get, respectively,

$$
\begin{aligned}
& \Delta\left(A_{11} X_{12}\right)=\Delta\left(A_{11} \circ X_{12}\right)=\Delta\left(A_{11}\right) \circ X_{12}+A_{11} \circ \Delta\left(X_{12}\right), \\
& \Delta\left(B_{11} X_{12}\right)=\Delta\left(B_{11} \circ X_{12}\right)=\Delta\left(B_{11}\right) \circ X_{12}+B_{11} \circ \Delta\left(X_{12}\right) .
\end{aligned}
$$

Therefore, it follows from Lemma 2 (iii) and equations (34)-(36) that

$$
\left(\Delta\left(A_{11}+B_{11}\right)-\Delta\left(A_{11}\right)-\Delta\left(B_{11}\right)\right) X_{12}+X_{12}\left(\Delta\left(A_{11}+B_{11}\right)-\Delta\left(A_{11}\right)-\Delta\left(B_{11}\right)\right)=0 .
$$

Multiplying equation (37) from the left by $P_{1}$ and from the right by $P_{2}$ and then by Lemma 1 (iv), we get

$P_{1}\left(\Delta\left(A_{11}+B_{11}\right)-\Delta\left(A_{11}\right)-\Delta\left(B_{11}\right)\right) P_{1} X_{12}=0$.

Therefore, by the faithfulness of $\mathscr{G}_{12}$, we get that $P_{1} \Delta\left(A_{11}+B_{11}\right) P_{1}=P_{1} \Delta\left(A_{11}\right) P_{1}+P_{1} \Delta\left(B_{11}\right) P_{1}$.
Hence, we get from equations (31)-(33) and (39) and Lemma 1 (iv) that $\Delta\left(A_{11}+B_{11}\right)=$ $\Delta\left(A_{11}\right)+\Delta\left(B_{11}\right)$. Similarly, we can show that $\Delta\left(A_{22}+B_{22}\right)=\Delta\left(A_{22}\right)+\Delta\left(B_{22}\right)$ holds for all $A_{22}, B_{22} \in \mathscr{G}_{22}$.

(vi) For any $A_{i i} \in \mathscr{G}_{i i}, A_{i j} \in \mathscr{G}_{i j}$, and $A_{j i} \in \mathscr{G}_{j i}$ $(1 \leq i \neq j \leq 2)$, it follows from $\mathscr{M} \mathscr{N}=\mathscr{N} \mathscr{M}=0$ that $\left[A_{i i}+A_{i j}+A_{j i}, P_{j}\right]=A_{i j}-A_{j i} \in \Omega$; taking $A=$ $A_{i i}+A_{i j}+A_{j i}, B=P_{j}$ in equation (8) and then by Lemma 1 (ii)-(iv) and Lemma 2 (iv), we obtain 


$$
\begin{aligned}
\Delta\left(A_{i j}\right)+\Delta\left(A_{j i}\right) & =\Delta\left(A_{i j}+A_{j i}\right) \\
& =\Delta\left(\left(A_{i i}+A_{i j}+A_{j i}\right) \circ P_{j}\right) \\
& =\Delta\left(A_{i i}+A_{i j}+A_{j i}\right) \circ P_{j}+\left(A_{i i}+A_{i j}+A_{j i}\right) \circ \Delta\left(P_{j}\right) \\
& =\Delta\left(A_{i i}+A_{i j}+A_{j i}\right) P_{j}+P_{j} \Delta\left(A_{i i}+A_{i j}+A_{j i}\right)+A_{i i} \Delta\left(P_{j}\right)+\Delta\left(P_{j}\right) A_{i i} \\
& =\Delta\left(A_{i i}+A_{i j}+A_{j i}\right) P_{j}+P_{j} \Delta\left(A_{i i}+A_{i j}+A_{j i}\right)-P_{i} \Delta\left(A_{i i}\right) P_{j}-P_{j} \Delta\left(A_{i i}\right) P_{i} .
\end{aligned}
$$

This implies that

$$
\begin{aligned}
& P_{i} \Delta\left(A_{i i}+A_{i j}+A_{j i}\right) P_{j}=P_{i} \Delta\left(A_{i i}\right) P_{j}+P_{i} \Delta\left(A_{i j}\right) P_{j}+P_{i} \Delta\left(A_{j i}\right) P_{j}, \\
& P_{j} \Delta\left(A_{i i}+A_{i j}+A_{j i}\right) P_{i}=P_{j} \Delta\left(A_{i i}\right) P_{i}+P_{j} \Delta\left(A_{i j}\right) P_{i}+P_{j} \Delta\left(A_{j i}\right) P_{i}, \\
& P_{j} \Delta\left(A_{i i}+A_{i j}+A_{j i}\right) P_{j}=0 .
\end{aligned}
$$

In the following, we show that $P_{i} \Delta\left(A_{i i}+A_{i j}\right.$ $\left.+A_{j i}\right) P_{i}=P_{i} \Delta\left(A_{i i}\right) P_{i}$. Indeed, for any $A_{11} \in \mathscr{G}_{11}$, $A_{12}, X_{12} \in \mathscr{G}_{12}, A_{21} \in \mathscr{G}_{21}$, on the one hand, it follows from $\mathscr{M} \mathscr{N}=\mathscr{N} \mathscr{M}=0$ that $\left[A_{11}+A_{12}+\right.$ $\left.A_{21}, X_{12}\right]=A_{11} X_{12}-X_{12} A_{21}+A_{21} X_{12}=A_{11} X_{12}$ $\in \Omega$; taking $A=A_{11}+A_{12}+A_{21}, B=X_{12}$ in equation (8) and so by $\mathscr{M} \mathscr{N}=\mathscr{N} \mathscr{M}=0$ and Lemma 1 (iii), we have

$$
\begin{aligned}
\Delta\left(A_{11} X_{12}\right)= & \Delta\left(\left(A_{11}+A_{12}+A_{21}\right) \circ X_{12}\right) \\
= & \Delta\left(A_{11}+A_{12}+A_{21}\right) \circ X_{12}+\left(A_{11}+A_{12}+A_{21}\right) \\
& \circ\left(X_{12}\right) \\
= & \Delta\left(A_{11}+A_{12}+A_{21}\right) \circ X_{12}+A_{11} \circ\left(X_{12}\right) .
\end{aligned}
$$

On the other hand, since $\left[A_{11}, X_{12}\right]=A_{11} X_{12} \in \Omega$, we get

$$
\begin{aligned}
\Delta\left(A_{11} X_{12}\right) & =\Delta\left(A_{11} \circ X_{12}\right) \\
& =\Delta\left(A_{11}\right) \circ X_{12}+A_{11} \circ\left(X_{12}\right) .
\end{aligned}
$$

Comparing equations (44) and (45), we get

$$
\left(\Delta\left(A_{11}+A_{12}+A_{21}\right)-\Delta\left(A_{11}\right)\right) X_{12}+X_{12}\left(\Delta\left(A_{11}+A_{12}+A_{21}\right)-\Delta\left(A_{11}\right)\right)=0 .
$$

Multiplying equation (46) from the left by $P_{1}$ and from the right by $P_{2}$ and then by Lemma 1 (iv), equation (43), and the faithfulness of $\mathscr{G}_{12}$, we get

$$
P_{1} \Delta\left(A_{11}+A_{12}+A_{21}\right) P_{1}=P_{1} \Delta\left(A_{11}\right) P_{1} .
$$

Similarly, for any $A_{22} \in \mathscr{G}_{22}, A_{12} \in \mathscr{G}_{12}$, and $A_{21} \in$ $\mathscr{G}_{21}$, we can get that

$$
P_{2} \Delta\left(A_{22}+A_{12}+A_{21}\right) P_{2}=P_{2} \Delta\left(A_{22}\right) P_{2} .
$$

Hence, for any $A_{i i} \in \mathscr{G}_{i i}, A_{i j} \in \mathscr{G}_{i j}$, and $A_{j i} \in \mathscr{G}_{j i}$ $(1 \leq i \neq j \leq 2)$, we get that

$$
P_{i} \Delta\left(A_{i i}+A_{i j}+A_{i j}\right) P_{i}=P_{i} \Delta\left(A_{i i}\right) P_{i} .
$$

Therefore, it follows from equations (41)-(48) and Lemma 1 (iii)-(iv) that $\Delta\left(A_{i i}+A_{i j}+A_{j i}\right)=$ $\Delta\left(A_{i i}\right)+\Delta\left(A_{i j}\right)+\Delta\left(A_{j i}\right)$.

(vii) For any $A_{i i} \in \mathscr{G}_{i i}, A_{i j} \in \mathscr{G}_{i j}, A_{j i} \in \mathscr{G}_{j i}$, and $A_{j j} \in$ $\mathscr{G}_{j j}(1 \leq i \neq j \leq 2)$, it follows from $\mathscr{M} \mathcal{N}=\mathscr{N} \mathscr{M}=0$ that $\quad\left[A_{i i}+A_{i j}+A_{j i}+A_{j j}, P_{j}\right]=A_{i j}-A_{j i} \in \Omega$; 
taking $A=A_{i i}+A_{i j}+A_{j i}+A_{j j}, B=P_{j}$ in equation (8) and so by Lemma 2 (vi) and Lemma 1 (iv), we have

$$
\begin{aligned}
\Delta\left(A_{i j}\right)+\Delta\left(A_{j i}\right)+2 \Delta\left(A_{j j}\right) & =\Delta\left(A_{i j}+A_{j i}+2 A_{j j}\right) \\
& =\Delta\left(\left(A_{i i}+A_{i j}+A_{j i}+A_{j j}\right) \circ P_{j}\right) \\
& =\Delta\left(A_{i i}+A_{i j}+A_{j i}+A_{j j}\right) \circ P_{j}+\left(A_{i i}+A_{i j}+A_{j i}+A_{j j}\right) \circ \Delta\left(P_{j}\right) \\
& =\Delta\left(A_{i i}+A_{i j}+A_{j i}+A_{j j}\right) \circ P_{j}+\left(A_{i i}+A_{j j}\right) \circ \Delta\left(P_{j}\right) .
\end{aligned}
$$

Similarly, we obtain

$$
2 \Delta\left(A_{i i}\right)+\Delta\left(A_{i j}\right)+\Delta\left(A_{j i}\right)=\Delta\left(A_{i i}+A_{i j}+A_{j i}+A_{j j}\right) \circ P_{i}+\left(A_{i i}+A_{j j}\right) \circ \Delta\left(P_{i}\right) .
$$

Therefore, it follows from (50) and (51), Lemma 1 (ii), and the property of 2-torsion freeness of $\mathscr{G}$ that $\Delta\left(A_{i i}+A_{i j}+\right.$ $\left.A_{j i}+A_{j j}\right)=\Delta\left(A_{i i}\right)+\Delta\left(A_{i j}\right)+\Delta\left(A_{j i}\right)+\Delta\left(A_{j j}\right)$. The proof is completed.

Lemma 3. $\Delta$ is an additive mapping.

$$
\begin{aligned}
\Delta(A+B) & =\Delta\left(\left(A_{11}+A_{12}+A_{21}+A_{22}\right)+\left(B_{11}+B_{12}+B_{21}+B_{22}\right)\right) \\
& =\Delta\left(\left(A_{11}+B_{11}\right)+\left(A_{12}+B_{12}\right)+\left(A_{21}+B_{21}\right)+\left(A_{22}+B_{22}\right)\right) \\
& =\Delta\left(A_{11}+B_{11}\right)+\Delta\left(A_{12}+B_{12}\right)+\Delta\left(A_{21}+B_{21}\right)+\Delta\left(A_{22}+B_{22}\right) \\
& =\Delta\left(A_{11}\right)+\Delta\left(B_{11}\right)+\Delta\left(A_{12}\right)+\Delta\left(B_{12}\right)+\Delta\left(A_{21}\right)+\Delta\left(B_{21}\right)+\Delta\left(A_{22}\right)+\Delta\left(B_{22}\right) \\
& =\Delta\left(A_{11}+A_{12}+A_{21}+A_{22}\right)+\Delta\left(B_{11}+B_{12}+B_{21}+B_{22}\right) \\
& =\Delta(A)+\Delta(B) .
\end{aligned}
$$

Therefore, $\Delta$ is an additive mapping on $\mathscr{G}$.The proof is completed.

Remark 1. For any $A \in \mathscr{G}$, we define a mapping $f: \mathscr{G} \longmapsto \mathscr{G}$ as

$$
f(A)=P_{2} \Delta\left(P_{1} A P_{2}\right) P_{1}+P_{1} \Delta\left(P_{2} A P_{1}\right) P_{2} .
$$

Then, by the definition of $f$, we can easily obtain that

$$
f\left(A_{i i}\right)=0 \text { and } f\left(A_{i j}\right)=P_{j} \Delta\left(A_{i j}\right) P_{i},
$$

for all $A_{i i} \in \mathscr{G}_{i i}, A_{i j} \in \mathscr{G}_{i j}(1 \leq i \neq j \leq 2)$.
Next, we will show that $f$ is an additive antiderivation on $\mathscr{G}$. First, we introduce Lemma 4 and get that $f$ is an additive mapping, and then, we introduce Lemmas 5 and 6 and show that $f$ is an additive antiderivation on $\mathscr{G}$.

Lemma 4. Let $f: \mathscr{G} \longmapsto \mathscr{G}$ be as in Remark 1. Then, $f$ is an additive mapping.

Proof. For any $A, B \in \mathscr{G}$, since we have shown that $\Delta$ is an additive mapping in Lemma 3 and then by the definition of $f$, we obtain that 


$$
\begin{aligned}
f(A+B) & =P_{2} \Delta\left(P_{1}(A+B) P_{2}\right) P_{1}+P_{1} \Delta\left(P_{2}(A+B) P_{1}\right) P_{2} \\
& =P_{2} \Delta\left(P_{1} A P_{2}+P_{1} B P_{2}\right) P_{1}+P_{1} \Delta\left(P_{2} A P_{1}+P_{2} B P_{1}\right) P_{2} \\
& =P_{2} \Delta\left(P_{1} A P_{2}\right) P_{1}+P_{2} \Delta\left(P_{1} B P_{2}\right) P_{1}+P_{1} \Delta\left(P_{2} A P_{1}\right) P_{2}+P_{1} \Delta\left(P_{2} B P_{1}\right) P_{2} \\
& =\left(P_{2} \Delta\left(P_{1} A P_{2}\right) P_{1}+P_{1} \Delta\left(P_{2} A P_{1}\right) P_{2}\right)+\left(P_{2} \Delta\left(P_{1} B P_{2}\right) P_{1}+P_{1} \Delta\left(P_{2} B P_{1}\right) P_{2}\right) \\
& =f(A)+f(B) .
\end{aligned}
$$

Therefore, $f$ is an additive mapping on $\mathscr{G}$.The proof is completed.

Lemma 5. Let $f: \mathscr{G} \longmapsto \mathscr{G}$ be as in Remark 1. Then, for any $A_{i i}, B_{i i} \in \mathscr{G}_{i i}, A_{i j}, B_{i j} \in \mathscr{G}_{i j}, B_{j i} \in \mathscr{G}_{j i}$, and $B_{j j} \in \mathscr{G}_{j j}(1 \leq i \neq$ $j \leq 2)$,

(i) $f\left(A_{i i} B_{i i}\right)=f\left(B_{i i}\right) A_{i i}+B_{i i} f\left(A_{i i}\right)$

(ii) $f\left(A_{i i} B_{j j}\right)=f\left(B_{j j}\right) A_{i i}+B_{j j} f\left(A_{i i}\right)$

(iii) $f\left(A_{i j} B_{j i}\right)=f\left(B_{j i}\right) A_{i j}+B_{j i} f\left(A_{i j}\right)$

(iv) $f\left(A_{i j} B_{i j}\right)=f\left(B_{i j}\right) A_{i j}+B_{i j} f\left(A_{i j}\right)$

(v) $f\left(A_{i i} B_{j i}\right)=f\left(B_{j i}\right) A_{i i}+B_{j i} f\left(A_{i i}\right)$

(vi) $f\left(A_{j i} B_{j j}\right)=f\left(B_{j j}\right) A_{j i}+B_{j j} f\left(A_{j i}\right)$

(vii) $f\left(A_{i i} B_{i j}\right)=f\left(B_{i j}\right) A_{i i}+B_{i j} f\left(A_{i i}\right)$

(viii) $f\left(A_{j i} B_{i i}\right)=f\left(B_{i i}\right) A_{j i}+B_{i i} f\left(A_{j i}\right)$

Proof

(i) For any $A_{i i}, B_{i i} \in \mathscr{G}_{i i}(1 \leq i \leq 2)$, it follows from $f\left(A_{i i} B_{i i}\right)=0=f\left(A_{i i}\right)=f\left(B_{i i}\right)$ that $f\left(A_{i i} B_{i i}\right)=$ $f\left(B_{i i}\right) A_{i i}+B_{i i} f\left(A_{i i}\right)$.

(ii) Similar to (i), we can showholds.

(iii) For any $A_{i j} \in \mathscr{G}_{i j}$ and $B_{j i} \in \mathscr{G}_{j i}(1 \leq i \neq j \leq 2)$, on the one hand, it follows from $\mathscr{M} \mathcal{N}=\mathscr{N} \mathscr{M}=0$ that $f\left(A_{i j} B_{j i}\right)=f(0)=0$. On the other hand, by the definition of $f$, we have $f\left(B_{j i}\right)=P_{i} \Delta\left(B_{j i}\right) P_{j}$ and $f\left(A_{i j}\right)=P_{j} \Delta\left(A_{i j}\right) P_{i}$ and so $f\left(B_{j i}\right) A_{i j}=0=$ $B_{j i} f\left(A_{i j}\right)$; therefore, we have $f\left(A_{i j} B_{j i}\right)=$ $f\left(B_{j i}\right) A_{i j}+B_{j i} f\left(A_{i j}\right)$.

(iv) For any $A_{i j}, B_{i j} \in \mathscr{G}_{i j}(1 \leq i \neq j \leq 2)$, on the one hand, we have $f\left(A_{i j} B_{i j}\right)=f(0)=0$. On the other hand, by the definition of $f$, we have $f\left(A_{i j}\right)=$ $P_{j} \Delta\left(A_{i j}\right) P_{i}$ and $f\left(B_{i j}\right)=P_{j} \Delta\left(B_{i j}\right) P_{i}$, and then, it follows from $\mathscr{M} \mathscr{N}=\mathscr{N} \mathscr{M}=0$ that $f\left(B_{i j}\right) A_{i j}=\left(P_{j}\right.$ $\left.\Delta\left(B_{i j}\right) P_{i}\right) A_{i j}=0$ and $B_{i j} f\left(A_{i j}\right)=B_{i j}\left(P_{j} \Delta\left(A_{i j}\right)\right.$ $\left.P_{i}\right)=0$ and so $f\left(A_{i j} B_{i j}\right)=f\left(B_{i j}\right) A_{i j}+B_{i j} f\left(A_{i j}\right)$.

(v) Similar to (iv), we can show that (v) holds.

(vi) Similar to (iv), we can show that (vi) holds.

(vii) For any $A_{i i} \in \mathscr{S}_{i i}$ and $B_{i j} \in \mathscr{S}_{i j}(1 \leq i \neq j \leq 2)$, since $\left[A_{i i}, B_{i j}\right]=A_{i i} B_{i j} \in \Omega$, we have

$$
\begin{aligned}
\Delta\left(A_{i i} B_{i j}\right) & =\Delta\left(A_{i i} \circ B_{i j}\right) \\
& =\Delta\left(A_{i i}\right) \circ B_{i j}+A_{i i} \circ \Delta\left(B_{i j}\right) \\
& =\Delta\left(A_{i i}\right) B_{i j}+B_{i j} \Delta\left(A_{i i}\right)+A_{i i} \Delta\left(B_{i j}\right)+\Delta\left(B_{i j}\right) A_{i i} .
\end{aligned}
$$

Hence, by the definition of $f$, we have

$$
f\left(A_{i i} B_{i j}\right)=P_{j} \Delta\left(A_{i i} B_{i j}\right) P_{i}=P_{j} \Delta\left(B_{i j}\right) A_{i i}=f\left(B_{i j}\right) A_{i i} .
$$

Therefore, we obtain from $f\left(A_{i i}\right)=0$ that $f\left(A_{i i} B_{i j}\right)=f\left(B_{i j}\right) A_{i i}+B_{i j} f\left(A_{i i}\right)$. Similarly, we can show (viii) holds. The proof is completed.

Lemma 6. Let $f: \mathscr{G} \longmapsto \mathscr{G}$ be as in Remark 3.1. Then, $f$ is an additive antiderivation.

Proof. For any $A, B \in \mathscr{G}$, let $A=A_{11}+A_{12}+A_{21}+A_{22}$ and $B=B_{11}+B_{12}+B_{21}+B_{22}$, where $A_{i j}, B_{i j} \in \mathscr{G}_{i j}(1 \leq i, j \leq 2)$, and we obtain from Lemmas 4 and 5 that

$$
\begin{aligned}
f(\mathrm{AB})= & f\left(\left(A_{11}+A_{12}+A_{21}+A_{22}\right)\left(B_{11}+B_{12}+B_{21}+B_{22}\right)\right) \\
= & f\left(A_{11} B_{11}\right)+f\left(A_{11} B_{12}\right)+f\left(A_{11} B_{21}\right)+f\left(A_{11} B_{22}\right)+f\left(A_{12} B_{11}\right)+f\left(A_{12} B_{12}\right)+f\left(A_{12} B_{21}\right)+f\left(A_{12} B_{22}\right)+f\left(A_{21} B_{11}\right) \\
& +f\left(A_{21} B_{12}\right)+f\left(A_{21} B_{21}\right)+f\left(A_{21} B_{22}\right)+f\left(A_{22} B_{11}\right)+f\left(A_{22} B_{12}\right)+f\left(A_{22} B_{21}\right)+f\left(A_{22} B_{22}\right)=f\left(B_{11}\right)\left(A_{11}+A_{12}+A_{21}+A_{22}\right) \\
& +f\left(B_{12}\right)\left(A_{11}+A_{12}+A_{21}+A_{22}\right)+f\left(B_{21}\right)\left(A_{11}+A_{12}+A_{21}+A_{22}\right)+f\left(B_{22}\right)\left(A_{11}+A_{12}+A_{21}+A_{22}\right) \\
& +\left(B_{11}+B_{12}+B_{21}+B_{22}\right) f\left(A_{11}\right) \\
& +\left(B_{11}+B_{12}+B_{21}+B_{22}\right) f\left(A_{12}\right)+\left(B_{11}+B_{12}+B_{21}+B_{22}\right) f\left(A_{21}\right)+\left(B_{11}+B_{12}+B_{21}+B_{22}\right) f\left(A_{22}\right)=f(B) A+B f(A) .
\end{aligned}
$$


Therefore, $f$ is an additive antiderivation on $\mathscr{G}$. The proof is completed.

Remark 2. For any $A \in \mathscr{G}$, we define a mapping $\delta: \mathscr{G} \longmapsto \mathscr{G}$ as

$$
\delta(A)=\Delta(A)-f(A) .
$$

Then, we obtain from Lemmas 3 and 4 that $\delta$ is an additive mapping on $\mathscr{G}$.

In the following, we will introduce Lemmas 7-9 and show that $\delta$ is an additive derivation on $\mathscr{G}$.

Lemma 7. Let $\delta: \mathscr{G} \longmapsto \mathscr{G}$ be as in Remark 2. Then, for any $A_{i i} \in \mathscr{G}_{i i}$ and $A_{i j} \in \mathscr{G}_{i j}(1 \leq i \neq j \leq 2)$,

(i) $\delta\left(P_{1}\right)=-\delta\left(P_{2}\right)$ and $\delta\left(P_{i}\right)=P_{i} \delta\left(P_{i}\right) P_{j}+P_{j} \delta\left(P_{i}\right) P_{i}$

(ii) $\delta\left(A_{i j}\right)=P_{i} \delta\left(A_{i j}\right) P_{j}$

(iii) $P_{j} \delta\left(A_{i i}\right) P_{j}=0, P_{i} \delta\left(A_{i i}\right) P_{j}=A_{i i} \delta\left(P_{i}\right) \quad$ and $P_{j} \delta\left(A_{i i}\right) P_{i}=\delta\left(P_{i}\right) A_{i i}$

Proof. By Lemma 1 and Remarks 1 and 2, we can easily check that Lemma 7 holds. The proof is completed.

Lemma 8. Let $\delta: \mathscr{G} \longmapsto \mathscr{G}$ be as in Remark 2. Then, for any $A_{i i}, B_{i i} \in \mathscr{G}_{i i}, A_{i j}, B_{i j} \in \mathscr{G}_{i j}, A_{j i}, B_{j i} \in \mathscr{G}_{j i}$, and $B_{j j} \in \mathscr{G}_{j j}(1$ $\leq i \neq j \leq 2$ ),

(i) $\delta\left(A_{i i} B_{i j}\right)=\delta\left(A_{i i}\right) B_{i j}+A_{i i} \delta\left(B_{i j}\right)$

(ii) $\delta\left(A_{i j} B_{j j}\right)=\delta\left(A_{i j}\right) B_{j j}+A_{i j} \delta\left(B_{j j}\right)$

(iii) $\delta\left(A_{i i} B_{i i}\right)=\delta\left(A_{i i}\right) B_{i i}+A_{i i} \delta\left(B_{i i}\right)$

(iv) $\delta\left(A_{i i} B_{j j}\right)=\delta\left(A_{i i}\right) B_{j j}+A_{i i} \delta\left(B_{j j}\right)$

(v) $\delta\left(A_{i j} B_{j i}\right)=\delta\left(A_{i j}\right) B_{j i}+A_{i j} \delta\left(B_{j i}\right)$

(vi) $\delta\left(A_{i j} B_{i j}\right)=\delta\left(A_{i j}\right) B_{i j}+A_{i j} \delta\left(B_{i j}\right)$

(vii) $\delta\left(A_{i i} B_{j i}\right)=\delta\left(A_{i i}\right) B_{j i}+A_{i i} \delta\left(B_{j i}\right)$ (viii) $\delta\left(A_{j i} B_{j j}\right)=\delta\left(A_{j i}\right) B_{j j}+A_{j i} \delta\left(B_{j j}\right)$

Proof

(i) For any $A_{i i}, B_{i j} \in \mathscr{G}_{i j}(1 \leq i \neq j \leq 2)$, on the one hand, since $\left[A_{i i}, B_{i j}\right]=A_{i i} B_{i j} \in \Omega$, we get that

$\Delta\left(A_{i i} B_{i j}\right)=\Delta\left(A_{i i}\right) B_{i j}+B_{i j} \Delta\left(A_{i i}\right)+A_{i i} \Delta\left(B_{i j}\right)+\Delta\left(B_{i j}\right) A_{i i}$.

And then, it follows from $\mathscr{M} \mathscr{N}=\mathscr{N} \mathscr{M}=0$, Lemma 1 , and Remark 1 that

$\Delta\left(A_{i i} B_{i j}\right)=\Delta\left(A_{i i}\right) B_{i j}+A_{i i} \Delta\left(B_{i j}\right)+f\left(B_{i j}\right) A_{i i}$.

On the other hand, we obtain from Lemma 6 and $f\left(A_{i i}\right)=0$ that

$$
f\left(A_{i i} B_{i j}\right)=f\left(B_{i j}\right) A_{i i} \text {. }
$$

Therefore, by Remark 2, equations (61) and (62), $A_{i i} f\left(B_{i j}\right)=A_{i i}\left(P_{j} \Delta\left(B_{i j}\right) P_{i}\right)=0$, and $f\left(A_{i i}\right)=0$, we get

$$
\begin{aligned}
\delta\left(A_{i i} B_{i j}\right) & =\Delta\left(A_{i i} B_{i j}\right)-f\left(A_{i i} B_{i j}\right) \\
& =\Delta\left(A_{i i}\right) B_{i j}+A_{i i} \Delta\left(B_{i j}\right) \\
& =\left(\Delta\left(A_{i i}\right)-f\left(A_{i i}\right)\right) B_{i j}+A_{i i}\left(\Delta\left(B_{i j}\right)-f\left(B_{i j}\right)\right) \\
& =\delta\left(A_{i i}\right) B_{i j}+A_{i i} \delta\left(B_{i j}\right) .
\end{aligned}
$$

(ii) Similarly, we can show that (ii) holds.

(iii) For any $A_{11}, B_{11} \in \mathscr{G}_{11}$ and $Y_{12} \in \mathscr{G}_{12}$, by Lemma 8 (i), on the one hand, we get

$$
\delta\left(A_{11} B_{11} Y_{12}\right)=\delta\left(\left(A_{11} B_{11}\right) Y_{12}\right)=\delta\left(A_{11} B_{11}\right) Y_{12}+A_{11} B_{11} \delta\left(Y_{12}\right) .
$$

On the other hand, we have

$$
\begin{gathered}
\delta\left(A_{11} B_{11} Y_{12}\right)=\delta\left(A_{11}\left(B_{11} Y_{12}\right)\right)=\delta\left(A_{11}\right) B_{11} Y_{12}+A_{11} \delta\left(B_{11}\right) Y_{12}+A_{11} B_{11} \delta\left(Y_{12}\right) . \\
P_{1} \delta\left(A_{11} B_{11}\right) P_{1}=P_{1} \delta\left(A_{11}\right) B_{11}+A_{11} \delta\left(B_{11}\right) P_{1} .
\end{gathered}
$$

Comparing equations (64) and (65), we get

$\left(\delta\left(A_{11} B_{11}\right)-\delta\left(A_{11}\right) B_{11}-A_{11} \delta\left(B_{11}\right)\right) Y_{12}=0$.
Furthermore, by Lemma 7 (i) and (iii), we have

This yields from the faithfulness of $\mathscr{G}_{12}$ that

$$
\begin{aligned}
P_{1} \delta\left(A_{11} B_{11}\right) P_{2}+P_{2} \delta\left(A_{11} B_{11}\right) P_{1} & =A_{11} B_{11} \delta\left(P_{1}\right)+\delta\left(P_{1}\right) A_{11} B_{11} \\
& =A_{11} \delta\left(B_{11}\right) P_{2}+P_{2} \delta\left(A_{11}\right) B_{11}
\end{aligned}
$$


Therefore, we obtain from Lemma 7 (iii) and equations (67) and (68) that

$$
\begin{aligned}
\delta\left(A_{11} B_{11}\right) & =P_{1} \delta\left(A_{11} B_{11}\right) P_{1}+P_{1} \delta\left(A_{11} B_{11}\right) P_{2}+P_{2} \delta\left(A_{11} B_{11}\right) P_{1} \\
& =P_{1} \delta\left(A_{11}\right) B_{11}+A_{11} \delta\left(B_{11}\right) P_{1}+A_{11} \delta\left(B_{11}\right) P_{2}+P_{2} \delta\left(A_{11}\right) B_{11} \\
& =\operatorname{delta}\left(A_{11}\right) B_{11}+A_{11} \delta\left(B_{11}\right) .
\end{aligned}
$$

Similarly, we can show $\delta\left(A_{22} B_{22}\right)=\delta\left(A_{22}\right) B_{22}+A_{22} \delta\left(B_{22}\right)$ holds for all $A_{22}, B_{22} \in \mathscr{G}_{22}$.

$$
\Delta\left(A_{i i}\right) B_{j j}+A_{i i} \Delta\left(B_{j j}\right) \in \mathscr{G}_{i j} \text { and } B_{j j} \Delta\left(A_{i i}\right)+\Delta\left(B_{j j}\right) A_{i i} \in \mathscr{G}_{j i} .
$$

Furthermore, since $\left[A_{i i}, B_{j j}\right]=0 \in \Omega$, we get

$$
\begin{aligned}
0 & =\Delta\left(A_{i i} \circ B_{j j}\right) \\
& =\Delta\left(A_{i i}\right) \circ B_{j j}+A_{i i} \circ \Delta\left(B_{j j}\right) \\
& =\Delta\left(A_{i i}\right) B_{j j}+B_{j j} \Delta\left(A_{i i}\right)+A_{i i} \Delta\left(B_{j j}\right)+\Delta\left(B_{j j}\right) A_{i i} \\
& =\left(\Delta\left(A_{i i}\right) B_{j j}+A_{i i} \Delta\left(B_{j j}\right)\right)+\left(B_{j j} \Delta\left(A_{i i}\right)+\Delta\left(B_{j j}\right) A_{i i}\right) .
\end{aligned}
$$

And so, this yields from equation (70) that

$$
\Delta\left(A_{i i}\right) B_{j j}+A_{i i} \Delta\left(B_{j j}\right)=0 .
$$

Therefore, it follows from equation (72) and $\delta\left(A_{i i} B_{j j}\right)=\delta(0)=0=f\left(A_{i i}\right)=f\left(B_{j j}\right)$ that

$$
\delta\left(A_{i i} B_{j j}\right)=\left(\Delta\left(A_{i i}\right)-f\left(A_{i i}\right)\right) B_{j j}+A_{i i}\left(\Delta\left(B_{j j}\right)-f\left(B_{j j}\right)\right)=\delta\left(A_{i i}\right) B_{j j}+A_{i i} \delta\left(B_{j j}\right) .
$$

(v) For any $A_{i j} \in \mathscr{G}_{i j}$ and $B_{j i} \in \mathscr{G}_{j i}(1 \leq i \neq j \leq 2)$, it

$$
\delta\left(A_{i j} B_{j i}\right)=\delta(0)=0 .
$$
follows from $\mathscr{M} \mathscr{N}=\mathscr{N} \mathscr{M}=0$ and Lemma 7 (ii) that

And,

$$
\delta\left(A_{i j}\right) B_{j i}=\left(P_{i} \delta\left(A_{i j}\right) P_{j}\right) B_{j i}=0 \text { and } A_{i j} \delta\left(B_{j i}\right)=A_{i j}\left(P_{j} \delta\left(B_{j i}\right) P_{i}\right)=0 .
$$

Thus, we obtain from (74) and (75) that

$$
\delta\left(A_{i j} B_{j i}\right)=\delta\left(A_{i j}\right) B_{j i}+A_{i j} \delta\left(B_{j i}\right) .
$$

(vi) Similar to (v), we can show that (vi) holds.

(vii) For any $A_{i i} \in \mathscr{S}_{i i}, A_{i j} \in \mathscr{S}_{i j}$, and $B_{j i} \in \mathscr{S}_{j i}(1 \leq i \neq$ $j \leq 2$ ), on the one hand, we get

$$
\delta\left(A_{i i} B_{j i}\right)=\delta(0)=0 .
$$

On the other hand, it follows from $\mathscr{M} \mathcal{N}=\mathscr{N} \mathscr{M}=$ 0 and Lemma 7 (ii)-(iii) that

$$
\begin{aligned}
\delta\left(A_{i i}\right) B_{j i} & =\left(P_{i} \delta\left(A_{i i}\right) P_{j}\right) B_{j i}=0 \text { and } A_{i i} \delta\left(B_{j i}\right) \\
& =A_{i i}\left(P_{j} \delta\left(B_{j i}\right) P_{i}\right)=0 .
\end{aligned}
$$

Comparing equations (77) and (78), we get

$$
\delta\left(A_{i i} B_{j i}\right)=\delta\left(A_{i i}\right) B_{j i}+A_{i i} \delta\left(B_{j i}\right) .
$$


(viii) Similar to (vii), we can show that (viii) holds. The proof is completed.

Lemma 9. Let $\delta: \mathscr{G} \longmapsto \mathscr{G}$ be as in Remark 2. Then, $\delta$ is an additive derivation.
Proof. For any $A, B \in \mathscr{G}$, let $A=A_{11}+A_{12}+A_{21}+A_{22}$ and $B=B_{11}+B_{12}+B_{21}+B_{22}$, where $A_{i j}, B_{i j} \in \mathscr{G}_{i j}(1 \leq i, j \leq 2)$; since $\delta$ is an additive mapping and then by Lemma 8 , we have

$$
\begin{aligned}
\delta(\mathrm{AB})= & \delta\left(\left(A_{11}+A_{12}+A_{21}+A_{22}\right)\left(B_{11}+B_{12}+B_{21}+B_{22}\right)\right) \\
= & \delta\left(A_{11} B_{11}\right)+\delta\left(A_{11} B_{12}\right)+\delta\left(A_{11} B_{21}\right)+\delta\left(A_{11} B_{22}\right)+\delta\left(A_{12} B_{11}\right)+\delta\left(A_{12} B_{12}\right)+\delta\left(A_{12} B_{21}\right)+\delta\left(A_{12} B_{22}\right)+\delta\left(A_{21} B_{11}\right) \\
& +\delta\left(A_{21} B_{12}\right)+\delta\left(A_{21} B_{21}\right)+\delta\left(A_{21} B_{22}\right)+\delta\left(A_{22} B_{11}\right) \\
& +\delta\left(A_{22} B_{12}\right)+\delta\left(A_{22} B_{21}\right)+\delta\left(A_{22} B_{22}\right)=\delta\left(A_{11}\right)\left(B_{11}+B_{12}+B_{21}+B_{22}\right)+\delta\left(A_{12}\right)\left(B_{11}+B_{12}+B_{21}+B_{22}\right) \\
& +\delta\left(A_{21}\right)\left(B_{11}+B_{12}+B_{21}+B_{22}\right)+\delta\left(A_{22}\right)\left(B_{11}+B_{12}+B_{21}+B_{22}\right) \\
& +\left(A_{11}+A_{12}+A_{21}+A_{22}\right) \delta\left(B_{11}\right)+\left(A_{11}+A_{12}+A_{21}+A_{22}\right) \delta\left(B_{12}\right)+\left(A_{11}+A_{12}+A_{21}+A_{22}\right) \delta\left(B_{21}\right) \\
& +\left(A_{11}+A_{12}+A_{21}+A_{22}\right) \delta\left(B_{22}\right)=\delta(A) B+A \delta(B) .
\end{aligned}
$$

Therefore, $\delta$ is an additive derivation on $\mathscr{G}$. The proof is completed.

Proof. of Theorem 1. For any $A \in \mathscr{G}$, let $A=A_{11}+A_{12}+A_{21}+A_{22}$, where $A_{i j} \in \mathscr{G}_{i j}(1 \leq i, j \leq 2)$; by Remarks 1 and 2 and Lemmas 6 and 9, we obtain that

$$
\Delta(A)=\delta(A)+f(A)
$$

where $\delta$ is an additive derivation and $f$ is an additive antiderivation, respectively. Furthermore, $f\left(A_{i i}\right)=0$ for all $A_{i i} \in \mathscr{G}_{i i}(1 \leq i \leq 2)$.

In the following, we check that $\delta$ and $f$ are unique. Let $d: \mathscr{G} \longmapsto \mathscr{G}$ be an additive derivation and $h: \mathscr{G} \longmapsto \mathscr{G}$ be an additive antiderivation such that $h\left(A_{i i}\right)=0$ for all $A_{i i} \in \mathscr{G}_{i i}$ $(1 \leq i \leq 2)$. Suppose that

$$
\Delta(A)=d(A)+h(A) .
$$

Then, for any $A \in \mathscr{G}$, by Theorem 1 , we get that $\delta(A)+$ $f(A)=\Delta(A)=d(A)+h(A)$ and so $\delta(A)-d(A)=h(A)-$ $f(A)$ for all $A \in \mathscr{G}$; this yields from $\delta$ and $d$ two additive derivations that $h-f$ is an additive derivation; moreover, by Remark 1, we get $(h-f)\left(A_{i i}\right)=0$ for all $A_{i i} \in \mathscr{G}_{i i}(1 \leq i \leq 2)$.

Next, we show that $(h-f)\left(A_{i j}\right)=0$ for all $A_{i j} \in \mathscr{G}_{i j}$ $(1 \leq i \neq j \leq 2)$. Indeed, for any $A_{i j} \in \mathscr{G}_{i j}(1 \leq i \neq j \leq 2)$, on the one hand, since $h-f$ is an additive derivation and $h\left(P_{i}\right)=f\left(P_{i}\right)=0(1 \leq i \leq 2)$ and so we get that

$$
\begin{aligned}
(h-f)\left(A_{i j}\right) & =(h-f)\left(P_{i} A_{i j}\right) \\
& =(h-f)\left(P_{i}\right) A_{i j}+P_{i}(h-f)\left(A_{i j}\right) \\
& =P_{i}(h-f)\left(A_{i j}\right) .
\end{aligned}
$$

On the other hand, since $h$ and $f$ are two additive antiderivations and $h\left(P_{j}\right)=f\left(P_{j}\right)=0(1 \leq j \leq 2)$, we get that

$$
\begin{aligned}
(h-f)\left(A_{i j}\right) & =(h-f)\left(A_{i j} P_{j}\right) \\
& =h\left(A_{i j} P_{j}\right)-f\left(A_{i j} P_{j}\right) \\
& =h\left(P_{j}\right) A_{i j}+P_{j} h\left(A_{i j}\right)-f\left(P_{j}\right) A_{i j}-P_{j} f\left(A_{i j}\right) \\
& =P_{j} h\left(A_{i j}\right)-P_{j} f\left(A_{i j}\right) \\
& =P_{j}(h-f)\left(A_{i j}\right) .
\end{aligned}
$$

Thus, this yields from equations (83) and (84) that $(h-$ f) $\left(A_{i j}\right)=0$ for all $A_{i j} \in \mathscr{G}_{i j}(1 \leq i \neq j \leq 2)$. Therefore, $(h-$ f) $(A)=(h-f) \quad\left(A_{11}\right)+(h-f)\left(A_{12}\right)+(h-f) \quad\left(A_{21}\right)+$ $(h-f)\left(A_{22}\right)=0$ and so $h=f$ and $\delta=d$. The proof is completed.

Remark 3. By the above lemmas, Remarks 1 and 2, and the proof of Theorem 1, we can easily obtain that if $\Delta$ is a nonlinear Jordan derivable mapping of $\mathscr{G}$ by Lie product square-zero elements, then the following statements are equivalent:

(i) $\Delta$ is an additive derivation

(ii) $P_{j} \Delta\left(A_{i j}\right) P_{i}=0, \forall A_{i j} \in \mathscr{G}_{i j}(1 \leq i \neq j \leq 2)$

(iii) $\Delta\left(A_{i j}\right) \in \mathscr{G}_{i j}, \forall A_{i j} \in \mathscr{G}_{i j}(1 \leq i \neq j \leq 2)$

Next, we give an application of Remark 3 to triangular algebras and we obtain that every nonlinear Jordan derivable mapping of a triangular algebra by Lie product square-zero elements is an additive derivation. 
Corollary 1. Let $\mathscr{A}$ and $\mathscr{B}$ be unital algebras, $\mathscr{M}$ be a unital $(\mathscr{A}, \mathscr{B})$-bimodule, which is faithful as both a left $\mathscr{A}$-module and a right $\mathscr{B}$-module, $\quad \mathcal{U}=\operatorname{Tri}(\mathscr{A}, \mathscr{M}, \mathscr{B})=$ $\left\{\left(\begin{array}{cc}a & m \\ 0 & b\end{array}\right): a \in \mathscr{A}, m \in \mathscr{M}, b \in \mathscr{B}\right\}$ be a 2-torsion free triangular algebra, and $\Delta: \mathcal{U} \longmapsto \mathcal{U}$ be a nonlinear Jordan derivable mapping of $\mathcal{U}$ by Lie product square-zero elements; then, $\Delta$ is an additive derivation.

Proof. of Corollary 1 . Let $1_{\mathscr{A}}$ and $1_{\mathscr{B}}$ be the identities of the algebras $\mathscr{A}$ and $\mathscr{B}$, respectively, and let 1 be the identity of the triangular algebra $\mathcal{U}$. We denote

$$
\begin{aligned}
& P_{1}=\left(\begin{array}{cc}
1_{\mathscr{A}} & 0 \\
0 & 0
\end{array}\right) \text { by the standard idempotent of } \mathcal{U}, \quad P_{2}=1-P_{1}=\left(\begin{array}{cc}
0 & 0 \\
0 & 1_{\mathscr{B}}
\end{array}\right), \\
& \mathscr{U}_{i j}=P_{i} \mathcal{U} P_{j} \text { for } 1 \leq i, j \leq 2 .
\end{aligned}
$$

It is clear that the triangular algebra $\mathcal{U}$ may be represented as

$$
\mathscr{U}=P_{1} \mathcal{U} P_{1}+P_{1} \mathscr{U} P_{2}+P_{2} \mathcal{U} P_{2}=\mathscr{A}+\mathscr{M}+\mathscr{B} .
$$

For any $A \in \mathcal{U}$, let $A=A_{11}+A_{12}+A_{22}$, where $A_{i j} \in \mathscr{U}_{i j}$ $(1 \leq i, j \leq 2)$; then for any $A_{12} \in \mathscr{U}_{12}$, since $\mathscr{U}_{21}=\{0\}$ and by Lemma 1 (iii), we get

$$
\Delta\left(A_{12}\right)=P_{1} \Delta\left(A_{12}\right) P_{2}+P_{2} \Delta\left(A_{12}\right) P_{1}=P_{1} \Delta\left(A_{12}\right) P_{2} .
$$

Therefore, we obtain from Remark 3 (iii) that $\Delta$ is an additive derivation. The proof is completed.

\section{Data Availability}

Data sharing is not applicable to this article as no datasets were generated or analysed during the current study.

\section{Conflicts of Interest}

The authors declare that they have no conflicts of interest.

\section{Authors' Contributions}

All authors contributed equally and significantly in this paper. All authors read and approved the final manuscript. This work was supported by the National Natural Science Foundation of China (Nos. 11901451 and 11901248).

\section{References}

[1] D. Benkovič, "Jordan derivations and antiderivations on triangular matrices," Linear Algebra and its Applications, vol. 397, no. 11, pp. 235-244, 2005.

[2] I. N. Herstein, "Jordan derivations of prime rings," Proceedings of the American Mathematical Society, vol. 8, no. 6, p. 1104, 1957.

[3] J. M. Cusack, "Jordan derivations on rings," Proceedings of the American Mathematical Society, vol. 53, no. 2, p. 321, 1975.

[4] M. Brešar and J. Vukman, "Jordan derivations on semiprime rings," Bulletin of the Australian Mathematical Society, vol. 37, no. 3, pp. 321-322, 1988.

[5] J. H. Zhang, "Jordan derivations on nest algebras," Acta Mathematica Sinica, vol. 41, no. 1, pp. 205-212, 1998.
[6] J.-H. Zhang and W.-Y. Yu, "Jordan derivations of triangular algebras," Linear Algebra and its Applications, vol. 419, no. 1, pp. 251-255, 2006.

[7] J. Zhu and C. Xiong, "All-derivable points in continuous nest algebras," Journal of Mathematical Analysis and Applications, vol. 340, no. 2, pp. 845-853, 2008.

[8] J. Zhao and J. Zhu, "Jordan higher all-derivable points in triangular algebras," Linear Algebra and its Applications, vol. 436, no. 9, pp. 3072-3086, 2012.

[9] W. B. Huang and J. K. Li, "Characterizations of Jordan mappings on some rings and algebras through zero products," Linear and Multilinear Algebra, vol. 60, no. 2, pp. 167-180, 2017.

[10] F. Lu, "Jordan derivable maps of prime rings," Communications in Algebra, vol. 38, no. 12, pp. 4430-4440, 2010.

[11] M. Ashraf and A. Jabeen, "Nonlinear Jordan triple derivable mappings of triangular algebras," Pacific Journal of Applied Mathematics, vol. 7, no. 4, pp. 229-239, 2016.

[12] Y. Li, L. V. Wyk, and F. Wei, "Jordan derivations and antiderivations of generalized matrix algebras," Operators and Matrices, vol. 7, no. 2, pp. 399-415, 2013.

[13] H. Ghahramani, "Jordan derivations on block upper triangular matrix algebras," 2013, https://arxiv.org/pdf/1312.6950.

[14] H. Ghahramani, "Jordan derivations on trivial extensions," Bulletin of the Iranian Mathematical Society, vol. 39, no. 4, pp. 635-645, 2013.

[15] W. S. Cheung, "Mappings on triangular algebras," Ph. D thesis, University of Victoria, Victoria, Canada, 2000.

[16] A. D. Sands, "Radicals and Morita contexts," Journal of Algebra, vol. 24, no. 2, pp. 335-345, 1973. 